\title{
Additional Esophagectomy Following Noncurative Endoscopic Resection for Esophageal Squamous Cell Carcinoma: is it a Reasonable Strategy?
}

\author{
Masayuki Watanabe, MD, PhD, FACS \\ Department of Gastroenterological Surgery, Cancer Institute Hospital of Japanese Foundation for Cancer Research, Tokyo, \\ Japan
}

Recent progress in endoscopic technology has enabled early detection of esophageal squamous cell carcinoma (ESCC). Endoscopic resection (ER), using endoscopic mucosal resection or endoscopic submucosal dissection techniques, has become standard for early-stage ESCC. Due to their lower invasiveness, the number of patients undergoing ER has been increasing worldwide. However, when pathologic diagnosis of an ER specimen suggests residual tumor or risk of lymph node metastasis, additional treatment is recommended.

Liu et al. conducted a retrospective review of cT1NOM0 ESCC patients who underwent noncurative ER followed by additional esophagectomy in eight tertiary-care centers in China. ${ }^{1}$ They reported that additional esophagectomy after noncurative ER allowed resection of residual tumor and nodal metastasis, with favorable outcomes. Recently, several authors have reported promising results of further treatment for patients after noncurative ER. ${ }^{2-6}$ This is the first multicenter study that included the most significant number of patients. I want to congratulate the authors for their excellent research.

Based on multivariate analysis to evaluate the risk of nodal metastasis, the authors suggested that submucosal invasion, lymphovascular invasion (LVI), and tumor size of $2 \mathrm{~cm}$ or more were the independent risk factors. ${ }^{1}$ The incidence of lymph node metastasis has been reported to

(C) Society of Surgical Oncology 2021

First Received: 9 July 2021

Accepted: 14 July 2021;

Published Online: 26 July 2021

M. Watanabe, MD, PhD, FACS

e-mail: masayuki.watanabe@jfcr.or.jp range from 8.3 to $53.1 \%$ in SM1 tumors (tumors limited to within one-third of the submucosal layer) and from 18.5 to $53.9 \%$ in SM2/3 tumors (tumors invading to deeper than two-thirds of the submucosal layer). ${ }^{7-9} \mathrm{We}$ previously reported that the incidence of lymph node metastasis was equivalent between additional esophagectomy after noncurative ER and upfront esophagectomy for cStage I ESCC. ${ }^{4}$ LVI is also known as a risk factor of lymph node metastasis. Eguchi et al. reported that the frequency of lymph node metastasis from SM1 ESCC with and without LVI was $28.6 \%$ and $100 \%$, respectively. ${ }^{7}$ Therefore, there seems to be no objection to the need for additional treatment in cases with submucosal invasion or with LVI.

On the contrary, there is no concrete evidence regarding the association between tumor size and lymph node metastasis in ESCC. In the Japanese guideline, ER is recommended for ESCC with major axis length of $5 \mathrm{~cm}$ or shorter from the viewpoint of post-ER stenosis ${ }^{10}$ but not on an oncological basis. Further research is needed to elucidate whether tumor length should be considered when deciding the indication for additional treatment.

Previous studies have reported the safety and oncologic efficacy of additional esophagectomy after noncurative ER. ${ }^{3,4,6}$ We noted that none of 57 patients who underwent additional esophagectomy after noncurative ER experienced recurrence. ${ }^{4}$ Similarly, Koterazawa et al. reported no recurrence among 28 patients who underwent esophagectomy in this situation. ${ }^{3}$ In the report of Liu et al., 4 of 128 patients $(3.1 \%)$ had tumor recurrence. ${ }^{1}$ We revealed that the number of metastatic lymph nodes was smaller in additional esophagectomy after noncurative ER than in upfront esophagectomy, although the distribution of metastatic lymph nodes was almost identical between the 
groups. $^{4}$ These findings suggest that additional esophagectomy is an oncologically practical choice after noncurative ER.

Chemoradiotherapy (CRT) is another option for additional treatment after noncurative ER. Compared with esophagectomy, CRT enables esophagus-preserving treatment. We have previously reported that overall and disease-specific survivals were comparable between patients treated with additional surgery and those who underwent CRT after noncurative ER. ${ }^{5}$ Meanwhile, disease recurrence was observed in 2 of 52 patients in the CRT group $(3.8 \%) .^{5}$ Miyata et al. also reported that disease recurrence was more often observed in the CRT group than in the surgery group. ${ }^{6}$ Although CRT may provide better quality of life (QOL) than surgery, additional CRT has a higher risk of disease recurrence to some extent.

Based on the accumulated experiences, the safety of ER has been improving. A Japanese clinical trial to confirm the efficacy of ER followed by CRT in patients with clinical SM1/SM2 cancer revealed that ER-related severe adverse events happened in only $0.6 \%$ of patients, suggesting the safety of ER for SM1/2 cancer. ${ }^{11}$ Meanwhile, accurate diagnosis of cancer invasion depth remains challenging, even when using image-enhanced magnifying endoscopy and endoscopic ultrasonography. Moreover, LVI is never diagnosed before the pathologic diagnosis. Therefore, ER can be used as a technique that enables total biopsy of superficial ESCC. By obtaining the pathologic findings of ER specimens, we can prevent unnecessary surgery. In the near future, genome-based individualized treatment based on ER pathology may be achieved.

Additional esophagectomy following noncurative ER is currently a reasonable strategy for early ESCC, with excellent oncologic safety. However, impairment of QOL after esophagectomy remains a significant problem for patients. Further research is needed to identify a group of patients with a low risk of lymph node metastasis and establish a watch-and-wait strategy.

DISCLOSURES The authors have nothing to disclose.

\section{REFERENCES}

1. Liu Z, Zhang J, Su Y, et al. Additional esophagectomy following noncurative endoscopic resection for early esophageal squamous cell carcinoma: a multicenter retrospective study. Ann Surg Oncol. 2021. https://doi.org/10.1245/s10434-021-10467-3.

2. Saeki H, Watanabe M, Mine S, et al. Esophagectomy for superficial esophageal cancer after non-curative endoscopic resection. J Gastroenterol. 2015;50:6-413.

3. Koterazawa Y, Nakamura T, Oshikiri T, et al. A comparison of the clinical outcomes of esophagectomy and chemoradiotherapy after noncurative endoscopic submucosal dissection for esophageal squamous cell carcinoma. Surg Today. 2018;48:783-9.

4. Maruyama S, Okamura A, Imamura Y, et al. Comparison of outcomes between additional esophagectomy after noncurative endoscopic resection and upfront esophagectomy for T1N0 esophageal squamous cell carcinoma. Ann Surg Oncol. 2021. h ttps://doi.org/10.1245/s10434-020-09498-z.

5. Kanie Y, Okamura A, Asari T, et al. Additional treatment following noncurative endoscopic resection for esophageal squamous cell carcinoma: a comparison of outcomes between esophagectomy and chemoradiotherapy. Ann Surg Oncol. 2021. h ttps://doi.org/10.1245/s10434-021-10225-5.

6. Miyata H, Sugimura K, Kanemura T, et al. Clinical outcomeof additional esophagectomy after endoscopic treatment for superficial esophageal cancer. Ann Surg Oncol. 2021. https://doi.org/ 10.1245/s10434-021-09864-5.

7. Eguchi T, Nakanishi Y, Shimoda T, et al. Histopathological criteria for additional treatment after endoscopic mucosal resection for esophageal cancer: analysis of 464 surgically resected cases. Mod Pathol. 2006;19:475-80.

8. Akutsu Y, Uesato M, Shuto K, et al. The overall prevalence of metastasis in T1 esophageal squamous cell carcinoma: a retrospective analysis of 295 patients. Ann Surg. 2013;257:1032-8.

9. Endo M, Yoshino K, Kawano T, et al. Clinicopathologic analysis of lymph node metastasis in surgically resected superficial cancer of the thoracic esophagus. Dis Esophagus. 2000;13:125-9.

10. Ishihara RR, Arima M, Iizuka T, et al. Endoscopic submucosal dissection/endoscopic mucosal resection guidelines for esophageal cancer. Dig Endosc. 2020;32:452-93.

11. Muto M, Minashi K, Nihei K, et al. Efficacy of combined endoscopic resection and chemoradiotherapy for clinical stage I esophageal squamous cell carcinoma (ESCC): a single-arm confirmatory study (JCOG0508). J Clin Oncol. 2016;34:4013-4013.

Publisher's Note Springer Nature remains neutral with regard to jurisdictional claims in published maps and institutional affiliations. 